\title{
Updated Distribution of Anopheline Mosquitoes (Diptera: Culicidae) in Hokkaido, Japan, and the First Evidence of Anopheles (Anopheles) Belenrae in Japan
}

Kyoko Sawabe ( $\nabla$ sawabe@nih.go.jp )

National Institute of Infectious Diseases https://orcid.org/0000-0002-6877-2010

Nozomi Imanishi-Kobayashi

Nagasaki University: Nagasaki Daigaku

Yoshihide Maekawa

National Institute of Infectious Diseases: Kokuritsu Kansensho Kenkyujo

\section{Yukiko Higa}

National Institute of Infectious Diseases: Kokuritsu Kansensho Kenkyujo

Kyeong Soon Kim

Tottori University: Tottori Daigaku

\section{Keita Hoshino}

National Institute of Infectious Diseases: Kokuritsu Kansensho Kenkyujo

\section{Yoshio Tsuda}

National Institute of Infectious Diseases: Kokuritsu Kansensho Kenkyujo

\section{Toshihiko Hayashi}

National Institute of Infectious Diseases: Kokuritsu Kansensho Kenkyujo

\section{Naoko Nihei}

National Institute of Infectious Diseases: Kokuritsu Kansensho Kenkyujo

Kenji Takai

Saint Marianna University School of Medicine: Sei Marianna Ika Daigaku

\section{Takeshi Kurihara}

National Institute of Infectious Diseases: Kokuritsu Kansensho Kenkyujo

Mutsuo Kobayashi

National Institute of Infectious Diseases: Kokuritsu Kansensho Kenkyujo

\section{Research}

Keywords: Anopheles hyrcanus group mosquitoes, Hokkaido Japan, ITS2 sequence

Posted Date: July 8th, 2021 
DOI: https://doi.org/10.21203/rs.3.rs-586010/v2

License: (c) (1) This work is licensed under a Creative Commons Attribution 4.0 International License. Read Full License 


\section{Abstract}

Background: In Hokkaido, northern island of Japan, at least seven cases of falciparum malaria were reported by 1951. A survey conducted at that time was unsuccessful in implicating any mosquito species as the possible vector. Although active anopheline mosquito surveillance continued until the middle of the 1980s, there is very limited information on their current status and distribution in Japan. Therefore, this study is an update on the current status and distribution of anopheline mosquitoes in Hokkaido based on a 15-year entomological surveillance between 2001 and 2015.

Methods: A survey of mosquitoes was conducted at 22 sites in Hokkaido, Japan, from 2001 to 2015. Adult mosquitoes were collected from cowsheds, lakesides, shrubs, and habitats ranging from open grassland to coniferous forest using a CDC miniature light trap enhanced with dry ice, aspirators, and sweeping nets. Larvae were collected from lakes, ponds, swamps, stagnant and flowing rivers, and paddy fields. All specimens were morphologically identified and subjected to PCR-based sequence analysis of the ITS2 region of rDNA. Phylogenetic trees were reconstructed using the neighbor-joining method.

Results: A total of 46 anopheline specimens were used for the phylogenetic analysis. During the survey, a new member of the Anopheles hyrcanus group, An. belenrae Rueda (2005), was discovered in eastern Hokkaido in 2004. Anopheles belenrae has since then been consistently found and confirmed to inhabit only this area of Japan. Four members of the An. hyrcanus group, An. belenrae, An. engarensis, An. lesteri, and An. sineroides, have been found in Hokkaido. The results also suggest that An. sinensis, formerly a dominant species throughout Japan, has become a rarely found species, at least currently in Hokkaido.

Conclusion: The updated distribution of anopheline mosquitoes in Hokkaido, Japan, showed considerable differences from that observed in previous surveys conducted from 1969 to 1984. In particular, areas where $A n$. sinensis was previously distributed may have been greatly reduced in Hokkaido. The phylogenetic analysis revealed a novel An. hyrcanus group member identified as $A n$. belenrae, described in South Korea in 2005. It is interesting that $A n$. belenrae was confirmed to inhabit only eastern Hokkaido, Japan.

\section{Background}

After World War II, both postwar malaria and endemic malaria were prevalent in many areas of Japan.

Reported cases reached 28,000 annually in 1945 and 1946, with over 7,000 cases of vivax malaria up to the end of the 1950s. Surprisingly, at least seven cases of falciparum malaria were reported between 1947 and 1951 in Rubeshibe, Hokkaido (43.78 N, 143.61 E), located in the north of Japan. Although a survey was conducted to determine the vector mosquitoes involved at that time, no suspected species were found [1]. Endemic malaria was considered eliminates by 1960 . The number of malaria cases has decreased drastically since then, with less than 80 imported cases annually in the past 10 years: 20 imported cases in 2020 [2]. 
Anopheline species contain the most important malaria vector species. Among those recorded in Japan, An. sinensis Wiedemann, 1828 is the most widespread and common anopheline species. This species is considered the major vector of vivax malaria in Korea and China. Previous surveys conducted in Japan revealed that $A n$. sinensis was the dominant anopheline species in Japan, including Hokkaido; An. lesteri Baisas \& Hu, 1936 was commonly found in Hokkaido with only a few An. sineroides Yamada, 1924 [3-6]. These surveys also found a new member of this group, An. engarensis Kanda and Oguma, 1978 [3-5]. Thus, several malaria vector species (e.g., An. sinensis, An. engarensis, and An. lesteri) continue to inhabit Japan. Despite the need for a nationwide survey to systematically assess these species, very little information is available, mostly gathered in the 1980s. Recently, several DNA barcoding projects have been conducted on mosquitoes in Japan, and a small number of genomic information on anopheline mosquitoes were included [7-9]. However, these studies were not specific to malaria vector mosquitoes.

At the onset of this survey, the distribution of five species of the An. hyrcanus group, An. sinensis, An. sineroides, An. lesteri, An. engarensis, and An. yatsushiroensis Miyazaki, 1951, had been confirmed in Japan. Moreover, of these five species, only An. yatsushiroensis has never been reported in Hokkaido [10-13], the region of interest in this study. Nonetheless, the highly similar morphological features of the members of this group, particularly An. engarensis and An. sinensis, makes it difficult to distinguish between species morphologically. Therefore, the frequency of clasper movements in males, hybridization studies, and chromosomal studies were used in distinguishing An. engarensis from the Japanese population of $A n$. sinensis [3-5]. Recently, they have effectively been identified using polymerase chain reaction (PCR) and sequence analysis. Among the molecular markers used for mosquito taxonomy, the cytochrome oxidase subunit 1 (COI) sequences of the DNA barcoding region [14-16], and the internal transcribed spacer 2 (ITS2) region of rDNA are the most efficient. ITS2 in particular, is very efficient in distinguishing between closely related species, e.g., An. maculipennis complex, An. quadrimaculatus complex, An. culicifacies complex, and An. gambiae complex [17-20]. ITS2 has also been used to address taxonomic issues in the An. hyrcanus group [21-26].

For about 20 years since the last survey in 1984 [6], very few surveys of malaria vector mosquitoes had been conducted in Japan. We therefore initiated nationwide surveys from 2001 to determine the current status and distribution of anopheline mosquitoes in Japan. During our survey, we recorded a new member of the An. hyrcanus group in eastern Hokkaido in 2004. They were genetically confirmed to be Anopheles (Anopheles) belenrae Rueda (2005), described in South Korea in 2005 [24]. In the present study, species identification and determination of genetic distances between specimens was carried out by analyzing ITS2 region. Special attention was given to determining the distribution of An. belenrae in Japan. Finally, we updated the information from previous surveys [3-6] on the current distribution of the anopheline mosquitoes in Hokkaido.

\section{Materials And Methods}

\section{Mosquito sampling}


Mosquitoes were collected at 22 sites in Hokkaido from 2001 to 2015 (Tables 1 and 2). In this study, eight specimens collected in domestic areas outside Hokkaido were used as a reference specimen in phylogenetic analysis. Seven of the eight specimens were from Japan and the last from Vietnam. The areas in Japan and year surveyed were Kanagawa Prefecture in 2001, Akita Prefecture in 2005, Aomori and Toyama Prefectures in 2007, and Gifu, Fukui and Tokushima Prefectures in 2009. The specimen collected in Vietnam, Gia Lai Province in 2007, served as an outside Japan An. sinensis reference strain. Details of the collection sites are provided in Table 2.

Adult mosquitoes were collected from cowsheds, lakesides, shrubs, and habitats ranging from open grassland to coniferous forest throughout the day using a CDC miniature light trap enhanced with dry ice [27], aspirators, and sweeping nets for approximately $3 \mathrm{~h}$ after sunset. Collected adult mosquitoes were frozen and transported in an icebox to the National Institute of Infectious Diseases (NIID), Tokyo, Japan. Larval mosquitoes were collected from paddy fields, swamps, stagnant and flowing rivers, lakes, and ponds using dippers. Larvae were transported alive to NIID and reared to adults under laboratory conditions of $25^{\circ} \mathrm{C}, 60-70 \%$ relative humidity, and a photoperiod of $16: 8$ (L:D) h. Morphological identification was performed on all adult individuals using taxonomic keys $[11,28]$. All classified mosquito specimens were transferred individually into $1.8 \mathrm{~mL}$ microtubes (Eppendorf, Hamburg, Germany), and stored at $-80^{\circ} \mathrm{C}$ until subsequent analyses by ITS 2 sequencing.

\section{DNA extraction, ITS2 amplification and sequencing}

Total genomic DNA was extracted from individual samples using a REDExtract-N-Amp Tissue PCR Kit (Sigma Chemical Co., St. Louis, MO) according to the manufacturer's protocol. Extracted mosquito DNA was subjected to PCR-based sequence analysis and phylogenetic analysis using primers of the ribosomal DNA ITS2 region (forward, 5'-TGT GAA CTG CAG GAC ACA-3'; reverse, 5'-TAT GCT TAA ATT CAG GGG GT3') [29]. Amplification conditions were as follows: initial denaturation at $95^{\circ} \mathrm{C}$ for $2 \mathrm{~min}$, followed by 35 cycles of $95^{\circ} \mathrm{C}$ for $30 \mathrm{~s}, 55^{\circ} \mathrm{C}$ for $30 \mathrm{~s}$, and $72^{\circ} \mathrm{C}$ for $1 \mathrm{~min}$, and a 4 min final extension at $72^{\circ} \mathrm{C}$ using a Veriti $^{\text {TM }}$ 96-well Thermal Cycler (Thermo Fisher Scientific Inc., Waltham, MA).

All visible PCR-amplified DNA fragments were purified using the Qiaquick PCR Purification Kit (QIAGEN, Venlo, Netherlands) or extracted using MonoFas (GL Sciences Inc., Tokyo, Japan) from a $2 \%$ low melting point agarose gel (SeaPlaque GTG agarose, Cambrex Corp., East Rutherford, NJ) after preparative gel electrophoresis and visualization with ethidium bromide. Each purified double-stranded PCR product was directly cycle-sequenced from both ends using a BigDye Terminator Cycle Sequencing FS Ready Reaction Kit v3.1 (Thermo Fisher Scientific Co.) and the PCR-primers [29]. The thermal profile used was 25 cycles of $96^{\circ} \mathrm{C}$ for $10 \mathrm{~s}, 55^{\circ} \mathrm{C}$ for $5 \mathrm{~s}$, and $60^{\circ} \mathrm{C}$ for 4 min using a Thermal Cycler, and ABI PRISM 3730 Genetic Analyzer (Thermo Fisher Scientific Co.).

Sequence analyses was performed using the GENETYX software ver. 14 (Genetyx Corp., Tokyo, Japan). Sequences of the PCR-amplified DNA fragments were then used to perform BLAST searches on the GenBank nucleic acid database (National Center for Biotechnology Information website, http://ww.ncbi.nIm.nih.gov/BLAST/) for species identification. 


\section{Phylogenetic analysis}

Multiple alignment of the ITS2 sequences with those of related species available in the GenBank library was performed using the CLUSTALW program [30]. Phylogenetic trees were produced using the neighborjoining (NJ) program with Kimura's two-parameter model [31] on MEGA X version 10.2.2 [32]. The statistical significance of the resulting $N J$ trees was evaluated using a bootstrap test with 1,000 replications.

Thirty-eight specimens (37 from Japan and one from Vietnam), made up of five species, out of the total collected mosquitoes were used for phylogenetic analyses. The corresponding sequence data for eight strains of six species: An. anthropophagus Xu and Feng strain SMMU-FK1 from China (GenBank accession no. AY803792) [33], An. belenrae strain isolate 3 from South Korea (AY375466) [34], An. kleini Rueda from South Korea (DQ177501, direct submission to the GenBank database), An. lesteri strain specimen 1 (type B) and specimen 2 (type C) from South Korea (AJ620899 and AJ620900, respectively) [25], An. sinensis strain isolate 1 from South Korea (AY375464) [34], and An. sineroides strain SINEK02 from South Korea (GU384724) [35], served as reference strains. Anopheles yatsushiroensis from China (AY186792) [22], which was renamed An. pullus M. Yamada [36, 37], was included as an outgroup sequence.

\section{Distribution map}

The distribution map was constructed using ArcGIS 10 (ESRI Inc., Redlans, CA), and information from the Geographic Information System.

\section{Results}

\section{Phylogenetic analysis}

A total of 248 specimens (181 adults and 67 larvae) were collected in Hokkaido between 2001 and 2015. The collected specimens were classified into four anopheline species of the An. hyrcanus group; $A n$. belenrae, An. engarensis, An. lesteri, and An. sineroides (Table 1). Interestingly, An. sinensis was not collected from Hokkaido during our survey. Phylogenetic analysis was performed using the 485bp ITS2 sequence of 38 specimens, collected from different sites (30 from Hokkaido, seven from Japanese regions outside Hokkaido, and one from Vietnam) in different years, and eight reference sequences from the GenBank database (Table 2).

The NJ phylogenetic trees revealed five robust clades, consisting of the four species listed above and $A n$. sinensis (Figs. 1 and 2). Unfortunately, An. sinensis was not detected in Hokkaido in this study. Therefore, we analyzed An. sinensis collected from areas outside Hokkaido. The cluster of An. sinensis showed small differences (Figs. 1 and 2). Nonetheless, there were no differences between the four Japanese strains of An. sinensis (Yokohama08, Echizen379, Kaifu353, and Misawa391) and the strain from South Korea (isolate 1). 
In 2004, two larvae morphologically identified as An. sinensis were confirmed to be An. belenrae using the ITS2 sequence, marking the first record of An. belenrae in Japan (Akan44 strain). Subsequent phylogenetic analysis showed that $A n$. belenrae was the closest related species to An. sinensis, followed by An. engarensis (Figs. 1 and 2).

\section{Intra- and interspecific ITS2 variation}

The levels of nucleotide variation detected between pairs of specimen in the An. hyrcanus group are presented in Table 3. There were no genetic differences between the 10 Japanese strains of An. belenrae (Akan44, Kushiro10, Kushiro201, Kushiro313, Kushiro418, Kawakami60, Akan712, Kushiro503, Nakagawa807, and Nakagawa26) and the Korean strain (isolate 3), with a $0 \%$ pairwise divergence (Figs. 1, 2 and Table 3). This suggests that the Japanese An. belenrae and the Korean An. belenrae are the same at least based on the ITS2 sequences. The Vietnamese strain (GLVN59) showed slight differences from the other strains, with a $0.26 \%$ pairwise divergence (Table 3 ). Regarding the clusters of An. engarensis, one specimen (Daisen55) collected in Akita Prefecture, an area outside Hokkaido, was slightly different from the nine specimens collected in Hokkaido (Abashiri02, Akashuri18, Yubari25, Yufutsu20, Kameda40, Yufutsu115, L1532, A14-22, and L1468) (Figs. 1 and 2). No genetic differences were observed among the An. engarensis specimens, except for the Daisen55 strain, with a $4.63 \%$ pairwise divergence from the others (Table 3 ).

As mentioned above, the intraspecific variation between these three species was very low $(0 \%, 0.26 \%$, and 4.63\% in An. belenrae, An. sinensis, and An. engarensis, respectively) (Table 3); a few differences were found based on the collection areas. Regarding the interspecific variation, pairwise divergence between An. belenrae and two other anopheline species ( $A n$. sinensis and An. engarensis) was $13.25-13.57 \%$ and 13.27-14.26\%, respectively, and 13.86-15.19\% between An. sinensis and An. engarensis (Table 3). The values among these three species indicate high levels of genetic differentiation. The NJ phylogenetic trees also showed that one strain of An. kleini from South Korea was located closer to An. engarensis than to An. belenrae and An. sinensis (Figs. 1 and 2). A detailed study of the genetic background of these species will be necessary.

In An. sineroides, no differences were found between the two specimens from Hokkaido (Fukagawa13 and Kushiro343), and the one from South Korea (SINEK02) (Figs. 1, 2, and Table 3). However, the two specimens from areas outside Hokkaido (Hida386 and Toyama80) showed few differences from the three mentioned above, with 0.26 and $0.8 \%$ pairwise divergences (Table 3 ). In contrast, a large intraspecific variation was observed in An. lesteri (Figs. 1 and 2). Pairwise divergence was in the range of 0.26-2.14\% among the nine strains from Hokkaido (Abashiri01, Abashiri17, Abashiri42, Hakodate31, Kamiiso35, Kameda38, Kushiro317, Kushiro505, and A2651) (Table 3). The two Korean strains (specimen 1 and specimen 2 classified as Type B and Type $\mathrm{C}$ of An. lesteri, respectively) were quite far from the other An. lesteri strains (Figs. 1 and 2). Pairwise divergence among the 11 An. lesteri strains ranged from $0.26-8.96 \%$, indicating that $A n$. lesteri appeared to form a highly divergent population. The cluster of $A n$. lesteri revealed a low pairwise divergence, ranging from $0-2.14 \%$, between the nine An. lesteri strains 
from Japan and the Chinese strain of An. anthropophagus (SMMU-FK1) (Table 3), suggesting that they may belong to the same species.

\section{Discussion}

\section{The first record of An. belenrae in Japan}

Our surveys from 2001 to 2015 revealed a significant change in the distribution range of the An. hyrcanus group in Hokkaido reported in the 1980s [3-6], including the first record of An. belenrae in Japan. Two larvae collected in the Kushiro Wetland in 2004 were tentatively named An. sinensis Kushiro strain, based solely on the morphological characteristics of the emerged adults. However, phylogenetic trees constructed using ITS2 sequence revealed that this An. sinensis Kushiro strain formed a robust clade that was clearly different from the clades of An. sinensis and other Anopheles species. Interestingly, the ITS2 sequence of the Kushiro strain was not identical to that of the An. sinensis strains collected in southern Japan, outside Hokkaido but to that of An. belenrae, a new strain reported in South Korea in 2005 [24]. The Kushiro strain could confidently be included in the An. belenrae cluster because of the absence of intraspecific divergence as mentioned above. This species was consistently found in the Kushiro Wetland after the first detection in 2004. In contrast, An. belenrae was not found outside Hokkaido in our 15-year nationwide survey. Thus, we concluded that this species is restricted to the Kushiro Wetland in Hokkaido.

The Kushiro Wetland is the largest marshland/wetland in Japan and is located in the Kushiro Plain. The Kushiro Wetland has been the focus of nature conservation efforts since before World War II, was registered as a Ramsar site in 1980 and designated as a national park in 1987. It is also famous for being the breeding ground for Japanese Cranes, Grus japonensis, and many other wild birds and a protected area for natural monuments, birds, and animals; thus, land development is strictly regulated. In South Korea, An. belenrae is found in the northern part of the country near the border with North Korea [24, 38, 39]. In China, An. belenrae is reportedly distributed in Shandong and Liaoning Provinces in northeast China, facing the Korean Peninsula [40]. These areas are not only geographically close to Japan, but may also have similarities in climate, vegetation, and some environmental factors with the Kushiro Wetland. However, further investigation is needed to compare the morphological characteristics of Japanese and Korean An. belenrae, and to determine the distribution of this species in locations outside Hokkaido in Japan. We hope that ecological and evolutionary factors impacting the emergence of An. belenrae will be elucidated with the development of molecular biological technology.

\section{No information of An. sinensis from Hokkaido}

The next noteworthy finding was the disappearance of An. sinensis from Hokkaido. In previous surveys, An. sinensis was generally distributed throughout Hokkaido [3-6] (Fig. 3). Although it is often found in the same larval habitat as An. lesteri, it is thought to occur more frequently in developed paddy fields and swamps [41]. In the 2000s, we did not find any An. sinensis in the habitat of An. lesteri, nor did we find any new sources or habitats (Fig. 4). It is possible that the larval habitat of An. sinensis changed drastically in the 20-year period between the previous studies [3-6] and this current study. For example, in 
the 1949 [1] and 1976 [6] surveys, four members of the An. hyrcanus group were detected in northeastern Hokkaido, around Rubeshibe (Fig. 3). At that time, there were paddy fields all over the district, and forestry and horse-logging were the main industries. In recent times however, the horse-logging industry has declined drastically, and the paddy fields have been replaced with upland crops. Furthermore, neither An. sinensis nor An. sineroides was found in this area, around Ozora, during our survey (Fig. 4). It is highly likely that the changes in vegetation and industry have affected the distribution of anopheline mosquitoes.

There may be other reasons for the disappearance of $A n$. sinensis from Hokkaido. The classification of organisms was mainly based on morphological keys until the 1990s. Although adults of An. belenrae can be separated morphologically from those of An. lesteri, An. sinensis and other species [24], it was likely that $A n$. belenrae and $A n$. sinensis could not be differentiated morphologically. Therefore, it should be noted that $A n$. belenrae may have been classified as An. sinensis. The results from the ITS 2 sequences in this study revealed that these two species were genetically the closest related. In addition, the pairwise interspecific distance in mitochondrial genomes calculated by each fragment showed minor or no difference between An. sinensis, An. belenrae and An. kleini [40]. Phylogenetic analysis of COI indicated that ancient hybridizations probably occurred among these three closely related species [42], making differentiation with the $\mathrm{COI}$ sequence improbable. To address this problem, we tried to extract DNA and decipher the nucleotide sequence from age-old, dried specimens previously classified as $A n$. sinensis collected in Hokkaido [3-6]. However, no new information could be obtained from these specimens. We hope that techniques for genetic analysis using age-old specimens will be developed as soon as possible.

Anopheles engarensis in Japan

Anopheles engarensis is also a species whose distributional range has reduced in Hokkaido. This species, first described in Engaro-cho (North-eastern Hokkaido) in 1977, [3] was also found in Monbetsu, Kushiro, and Obihiro until 1984 [6], suggesting a wide distribution in Hokkaido [3-6] (Fig. 3). However, our surveillance found this species to be restricted to western and southern Hokkaido (Fig. 4). In addition, this species was also collected in northern Tohoku, Akita Prefecture, suggesting a southward shift presumably due to changes in the environment, including the climate of larval habitats. In terms of classification, An. engarensis was recognized as a new species in the An. hyrcanus group only after its chromosomal structure was determined to be different from An. sinensis [4]. This was because of the high morphological similarity between the two species. Indeed, the only distinguishing feature was the unique number of clasper movements of An. engarensis males during artificial mating, a common method for laboratory maintenance of anopheline mosquitoes [5].

In general, ITS2 is known to have high interspecific and low intraspecific variability; however, extensive intraspecific variations have been reported in anopheline mosquitoes. For instance, ITS2 intraspecific variations ranged from $0.2-19.0 \%$ for the Latin American anophelines [43]. In the An. hyrcanus group, the average intraspecific distance was $0.3 \%$, but no intraspecific variations were observed in An. belenrae [42]. These results suggest that the ITS2 spacer is a good marker for differentiating between members of 
the An. hyrcanus group. In this study, there were no intraspecific variations in the An. belenrae, $A n$. engarensis, and $A n$. sineroides strains from Hokkaido. However, there was significant intraspecific variation between the nine An. engarensis strains from Hokkaido and the Daisen 55 strain from Akita Prefecture. The genetic distance of $4.7 \%$ was considerably greater than the $0.22 \%$ intraspecific variation in the An. sinensis strains from Vietnam, South Korea, and Japan. We inferred that the Daisen55 An. engarensis strain was not introduced from Hokkaido but inhabited the Tohoku region independently. On the other hand, in species groups consisting of recently diverged members, such as the An. gambiae complex, the interspecific differences in ITS2 were reported to be minor, ranging from $0.4-1.6 \%$ [20]. It is possible that $A n$. engarensis is a recently diverged lineage.

Anopheles lesteri in Hokkaido

In Rubeshibe Hokkaido, at least seven cases of falciparum malaria were recorded between 1946 and 1947. An unsuccessful survey was conducted to determine the vector mosquitoes involved in the transmission although An. sinensis and An. sineroides were collected [1]. During the falciparum malaria epidemic in the vicinity of Guangdong City, China, around 1942, the transmission was inferred to have involved An. lesteri and not $A n$. sinensis. This inference was based on results from field investigations and subsequent infection experiments with Plasmodium falciparum [44]. Based on this inference, it was suggested but never confirmed that An. lesteri may have been involved in the outbreak of falciparum malaria in Rubeshibe, Hokkaido. In terms of distribution, An. lesteri which was initially thought to be restricted to western Islands of Japan, such as the Kyushu Island [44], was also found in various areas of Honshu mainland of Japan [12], Hokkaido [45], Okinawa Island, and Yaeyama Islands [46]. The present survey confirmed that An. lesteri is still widely distributed in Hokkaido (Figs. 3 and 4). At the start of our survey in 2001, we noticed female mosquitoes collected in Ozora, Hokkaido, to have an intense affinity for human blood. These female mosquitoes were therefore considered, and subsequently confirmed, to be $A n$. lesteri based on the reported high anthropophilic nature of An. lesteri relative to An. sinensis and other members of the An. hyrcanus group $[12,47]$. We therefore expected to easily collect An. lesteri in subsequent surveys in Hokkaido.

In our study, the ITS2 intraspecific distance in An. lesteri ranged from $0-9.44 \%$. These values suggest that An. lesteri is a highly divergent species when An. lesteri type B (specimen 1) and type C (specimen 2) from South Korea are included in this species. Since the ITS2 distance of this species varies even within Hokkaido, there is a possibility that An. lesteri includes crypto-species. In defining this species, it is necessary to analyze both the COI barcoding region and the ITS2 region. Moreover, a large number of specimens, collected outside Hokkaido, will be necessary. In a previous study, a short interspecific distance of $7.2 \%$ was observed between An. kleini and An. engarensis [42]. We obtained similar ITS2 distances of $6.47 \%$ and $8.67 \%$ between An. kleini and our An. engarensis specimens from Hokkaido and $A n$. Kleini and An. engarensis Daisen55 strain, respectively. Although these results may provide validation that $A n$. Kleini is a synonym of $A n$. engarensis, further analysis is required. We also presented evidence that An. anthropophagus and An. lesteri were conspecific, based on the ITS2 divergence between them. Our results based on interspecific comparisons of ITS2 divergence may also support previous reports that 
An. belenrae and $A n$. sinensis are genetically distinct [24, 25], and An. anthropophagus is a conspecific species of An. lesteri [34, 48].

\section{Conclusions}

ITS2 sequence divergence clearly disclosed the current distribution of the An. hyrcanus group mosquitoes in Hokkaido, demonstrating great differences from surveys conducted between 1969 and 1984. In particular, the area inhabited by $A n$. sinensis has greatly reduced, and the newly discovered $A n$. belenrae was confirmed to inhabit only eastern Hokkaido. In summary, this study showed that Hokkaido harbored four members of the An. hyrcanus group, namely An. engarensis, An. belenrae, An. sineroides, and An. lesteri.

Here, we have demonstrated the presence of two anopheline species that have been reported as malaria vectors, An. belenrae instead of An. sinensis, in addition to An. lesteri, in Hokkaido since 2001. Although the malaria vector capacity of the Japanese strain of An. belenrae has not yet been evaluated, the Korean strain is considered to be a vector or potential vector of $P$. vivax $[24,49]$. Fortunately, all recently reported cases of malaria in Japan have been imported. However, emergence of potential autochthonous malaria epidemics should always be of concern because multiple malaria vector species still remain in Japan, as confirmed in this study.

\section{Abbreviations}

NIID: National Institute of Infectious Diseases; ITS2: Internal Transcribed spacer 2; COI: cytochrome oxidase subunit 1

\section{Declarations}

\section{Ethics approval and consent to participate}

Not applicable.

\section{Consent for publication}

Not applicable.

\section{Availability of data and materials}

All data generated or analyzed during this study are included in this published article. The newly generated sequences were submitted in the GenBank database under the accession numbers LC634739LC634772.

\section{Competing interests}


The authors declare that they have no competing interests.

\section{Funding}

This work was supported by grant-in-aids awarded by the Ministry of Health, Labor, and Welfare $(\mathrm{H} 18$ Shinko-Ippan-009, H21-Shinko-Ippan-005 and H24-Shinko-Ippan-007), the Research Program on Emerging and Re-emerging Infectious Diseases from Japan Agency for Medical Research and Development (AMED) (JP20fk0108067 and JP21fk0108613), and by Japan Initiative for Global Research Network on Infectious Diseases (J-GRID) from Ministry of Education, Culture, Sports, Science and Technology in Japan and AMED (JP17fm0108018).

\section{Author's contributions}

$\mathrm{KS}, \mathrm{TK}, \mathrm{KT}, \mathrm{NN}$ and MK conceived and designed this study. KS, NI and YM drafted the manuscript. KS and $\mathrm{NI}$ conducted phylogenetic analyses. YM and NN analyzed GIS information and drew the distibution maps. YH, KSK, KT, YT and TH contributed to facilitation in field invstigations. All authors participated in field investigations. All authors contributed to revise the manuscript and approved the final manuscript.

\section{Acknowledgements}

We are deeply grateful to the late Dr. Takashi Kurihara for much support, encouragement and scientific advice in our research on malaria vectors in Asia. If he had been alive, he should have been a cocorresponding author.

We express our thanks to Dr. Leopold M. Rueda, for his valuable taxonomical and ecological information of An. belenrae. We also thank Drs. Mamoru Watanabe, Tran Vu Phong, Nguyen Thi Yen, Michael AmoaBosompem, and the staff members of the Department of Medical Entomology of NIID, for their kind making of the arrangements for the field investigations and also for their helpful suggestions and critiques. We would like to thank Editage (www.editage.com) for English language editing.

\section{References}

1. Sasa M, Takahashi H, Kohgo T, Oshima H. An epidemic of the falciparum malaria in Hokkaido. Sogo Igaku. 1949;6:340-3. (In Japanese).

2. Infectious Diseases Weekly Report (IDWR), IDWR surveillance data table 2020 week 53. National Institute of Infectious Diseases, Japan. 2020. https://www.niid.go.jp/niid/ja/data/10103-idwrsokuho-data-j-2053.html. Accessed 01 May, 2021.

3. Oguma M, Kanda T. The distribution of Anopheles sinensis, A. sinensis "E" A. lesteri and A. sineroides at thirty-four localities of Japan. Jpn J Sanit Zool. 1977;28:417 - 21.

4. Kanda T, Oguma Y. Anopheles engarensis, a new species related to sinensis from Hokkaido Island, Japan. Mosg Syst. 1978;10:45-53. 
5. Kanda T, Oguma Y. Morphological variations of Anopheles sinensis Wiedemann, 1828 and A. lesteri Baisas and Hu, 1936 and frequency of clasper movements of the males of several Anopheles species during induced copulation. Jpn J Sanit Zool. 1976;27:325 - 31.

6. Nagashima Y. The research for Anopheles (Anopheles) sinensis group with reference to epidemiological aspects of malaria in Japan. Niigata Med J. 1986;100:264-86. (In Japanese with English summary).

7. Taira K, Toma T, Tamashiro M, Miyagi I. DNA barcoding for identication of mosquitoes (Diptera: Culicidae) from the Ryukyu Archipelago, Japan. Med Entomol Zool. 2012;63:289-306.

8. Maekawa Y, Tsuda Y, Sawabe K. A nationwide survey on distribution of mosquitoes in Japan. Med Entomol Zool. 2016;1:1-12. (In Japanese with English summary).

9. Maekawa Y, Ogawa K, Komagata O, Tsuda Y, Sawabe K. DNA barcording for molecular identification of Japanese mosquitoes. Med Entomol Zool. 2016;7:183-98. (In Japanese with English summary).

10. Reid JA. The Anopheles Hyrcanus group in South-East Asia. Bull Ent Res. 1953;44:5-76.

11. Tanaka K, Mizusawa K, Saugustad ES. A revision of the adult and larval mosquitoes of Japan (including the Ryukyu archipelago and the Ogasawara Islands) and Korea (Diptera: Culicidae). Contr Am Entomol Inst. 1979;16:1-987.

12. Otsuru M, Ohmori N. Malaria studies in Japan after World War II. Part II. The research for Anopheles sinensis sibling species group. Jpn J Exp Med. 1960;30:33-65.

13. Harrison BA. A lectotype designation and description for Anopheles (An.) sinensis Wiedemann I828, with a discussion of the classification and vector status of this and some other oriental Anopheles. Mosg Syst. 1973;5:1-13.

14. Folmer O, Black M, Hoeh W, Lutz R, Vrijenhoek R. DNA primers for amplication of mitochondrial cytochrome coxidase subunit I from diverse metazoan invertebrates. Mol Mar Biol Biotechnol. 1994;3:294-9.

15. Hebert PD, Cywinska A, Ball SL, Waard JR. Biological identifications through DNA barcodes. Proc Biol Sci. 2003;70:313-21.

16. Hebert PD, Penton EH, Burns JM, Janzen DH, Hallwachs W. Ten species in one: DNA barcoding reveals cryptic species in the neotropical skipper butterfly Astraptes fulgerator. Proc Natl Acad Sci USA. 2004;101:14812-7.

17. Proft J, Maier WA, Kampen $\mathrm{H}$. Identification of six sibling species of the Anopheles maculipennis complex (Diptera: Culicidae) by a polymerase chain reaction assay. Parasitol Res. 1999;85:837-43.

18. Cornel AJ, Porter $\mathrm{CH}$, Collins FH. Polymerase chain reaction species diagnostic assay for Anopheles quadrimaculatus cryptic species (Diptera: Culicidae) based on ribosomal DNA ITS2 sequences. J Med Entomol. 1996;33:109-16.

19. Goswami G, Raghavendra K, Nanda N, Gakhar SK, Subbarao SK. PCR-RFLP of mitochondrial cytochrome oxidase subunit II and ITS2 of ribosomal DNA: markers for the identification of members of the Anopheles culicifacies complex (Diptera: Culicidae). Acta Trop. 2005;95:92-9. 
20. Paskewitz SM, Wesson DM, Collins FH. The internal transcribed spacers of ribosomal DNA in five members of the Anopheles gambiae species complex. Insect Mol Biol. 1993;2:247-57.

21. Gao Q, Beebe NW, Cooper RD. Molecular identification of the malaria vectors Anopheles anthropophagus and Anopheles sinensis (Diptera: Culicidae) in central China using polymerase chain reaction and appraisal of their position within the Hyrcanus Group. J Med Entomol. 2004;41:5-11.

22. Yajun M, Xu J. The Hyrcanus group of Anopheles (Anopheles) in China (Diptera: Culicidae): species discrimination and phylogenetic relationships inferred by ribosomal DNA internal transcribed spacer 2 sequences. J Med Entomol. 2005;42:610-9.

23. Li C, Lee JS, Groebner JL, Kim HC, Klein TA, O'Guinn ML, Wilkerson RC. A newly recognized species in the Anopheles Hyrcanus group and molecular identification of related species from the Republic of South Korea (Diptera: Culicidae). Zootaxa. 2005;939:1-8.

24. Rueda LM. Two new species of Anopheles (Anopheles) Hyrcanus Group (Diptera: Culicidae) from the Republic of South Korea. Zootaxa. 2005;941:1-26.

25. Hwang UW. Revisited ITS2 phylogeny of Anopheles (Anopheles) Hyrcanus group mosquitoes: reexamination of unidentified and misidentified ITS2 sequences. Parasitol Res. 2007;101:885-94.

26. Djadid ND, Jazayeri H, Gholizadeh S, Rad SP, Zakeri S. First record of a new member of Anopheles Hyrcanus Group from Iran: molecular identification, diagnosis, phylogeny, status of kdr resistance and Plasmodium infection. J Med Entomol. 2009;46:1084-93.

27. Tsuda Y, Higa Y, Kurahashi H, Hayashi T, Hoshino K, Komagata O, Isawa H, Kasai S, Sasaki T, Tomita T, Sawabe K, Nihei N, Kobayashi M. Dry-ice trap collection of mosquitoes at urban areas surrounding Tokyo, Japan in 2003 and 2004. Med Entomol Zool. 2006;57:75-82. (Japanese with English Summary).

28. Stojanovich CJ, Scott HG. Illustrated Key to Mosquitoes of Vietnam. Atlanta, GA: U. S. Department of Health, Education, and Welfare, Public Health Service. 1966.

29. Beebe NW, Saul A. Discrimination of all members of the Anopheles punctulatus complex by polymerase chain reaction-restriction fragment length polymorphism analysis. Am J Trop Med Hyg. 1995;53:478-81.

30. Thompson JD, Higgins DG, Gibson TJ. CLUSTAL W: Improving the sensitivity of progressive multiple sequence alignment through sequence weighting, position-specific gap penalties and weight matrix choice. Nucleic Acids Res. 1994;22:4673-80.

31. Kimura M. A simple method for estimating evolutionary rates of base substitutions through comparative studies of nucleotide sequences. J Mol Evol. 1980;16:111-20.

32. Kumar S, Stecher G, Li M, Knyaz C, Tamura K. MEGA X: Molecular Evolutionary Genetics Analysis across Computing Platforms. Mol Biol Evol. 2018;35:1547-9.

33. Ma Y, Yang P. Taxonomic study on Anopheles anthropophagus from China (Diptera: Culicidae): inferred by morphology, chromosome karyotype and molecular markers. Kun Chong Fen Lei Xue Bao. 2005;27:199-208. (In Chinese with English Summary). 
34. Wilkerson RC, Li C, Rueda LM, Kim HC, Klein TA, Song GH, Strickman D. Molecular confirmation of Anopheles (Anopheles) lesteri from the Republic of South Korea and its genetic identity with $A n$. (Ano.) anthropophagus from China (Diptera: Culicidae). Zootaxa. 2003;378:1-14.

35. Joshi D, Park MH, Saeung A, Choochote W, Min GS. Multiplex assay to identify Korean vectors of malaria. Mol Ecol Resour. 2010;10:748-50.

36. Shin E, Hong HK. A new synonym of Anopheles (Anopheles) pullus Yamada, 1937: A. (A.) yatsushiroensis Miyazaki, 1951. Korean J Appl Entomol. 2001;31:1-5.

37. Hwang UW, Yong TS, Ree H. Molecular evidence for synonymy of Anopheles yatsushiroensis and An. pullus. J Am Mosq Control Assoc. 2004;20:99-104.

38. Rueda LM, Kim HC, Klein TA, Pecor JE, Li C, Sithiprasasna R, Debbouns M, Wilkerson RC. Distribution and larval habitat characteristics of Anopheles Hyrcanus group and related mosquito species (Diptera: Culicidae) in South Korea. J Vector Ecol. 2006;31:199-206.

39. Kim HC, Klein TA, Lee WJ. Mosquito species distribution and larval breeding habitats with taxonomic identification of Anopheline mosquitoes in Korea. Entomol Res. 2007;37:29-35.

40. Zhu HM, Luo SH, Gao M, Tao F, Gao JP, Chen HM, Li XY, Peng H, Ma YJ. Phylogeny of certain members of Hyrcanus group (Diptera: Culicidae) in China based on mitochondrial genome fragments. Infect Dis Poverty. 2019;8:91-7.

41. Kurihara T. Malaria vectors in Japan (except Nansei Islands). Med Entomol Zool. 2002;53:1-28. (In Japanese with English summary).

42. Fang Y, Shi WQ, Zhang Y. Molecular phylogeny of Anopheles hyrcanus group members based on ITS2 rDNA. Parasites Vectors. 2017; 10:417-27.

43. Marrelli MT, Sallum MAM, Marinotti 0 . The second internal transcribed spacer of nuclear ribosomal DNA as a tool for Latin American anopheline taxonomy - a critical review. Mem Inst Oswaldo Cruz. 2006;101:817-32.

44. Otsuru M. On a new type of Anopheles hyrcan from Japan. Fukuoka Med J. 1949;40:139-48. (In Japanese).

45. Kamimura K. The distribution and habit of medically important mosquitoes of Japan. Med Entomol Zool. 1968;19:15-34. (In Japanese with English summary).

46. Tanaka K. Mosquitoes of the Ryukyu Archipelago (Diptera: Culicidae). Mosq Syst. 1975;7:207-33.

47. Ho C, Chou TC, Chen TH, Hseuh AT. The Anopheles hyrcanus group and its relation to malaria in East China. Chin Med J. 1962;81:71-8.

48. Hwang UW, Tang LH, Kobayashi M, Yong TS, Ree HII. Molecular evidence supports that Anopheles anthropophagus from China and Anopheles lesteri from Japan are the same species. J Am Mosq Control Assoc. 2006;22:324-6.

49. Rueda LM, Li C, Kim HC, Klein TA, Foley DH, Wilkerson RC. Anopheles belenrae, a potential vector of Plasmodium vivax in the Republic of Korea. J Am Mosq Contol Assoc. 2010;26:430-2. 


\section{Tables}

Due to technical limitations, table 1 to 3 is only available as a download in the Supplemental Files section.

\section{Figures}

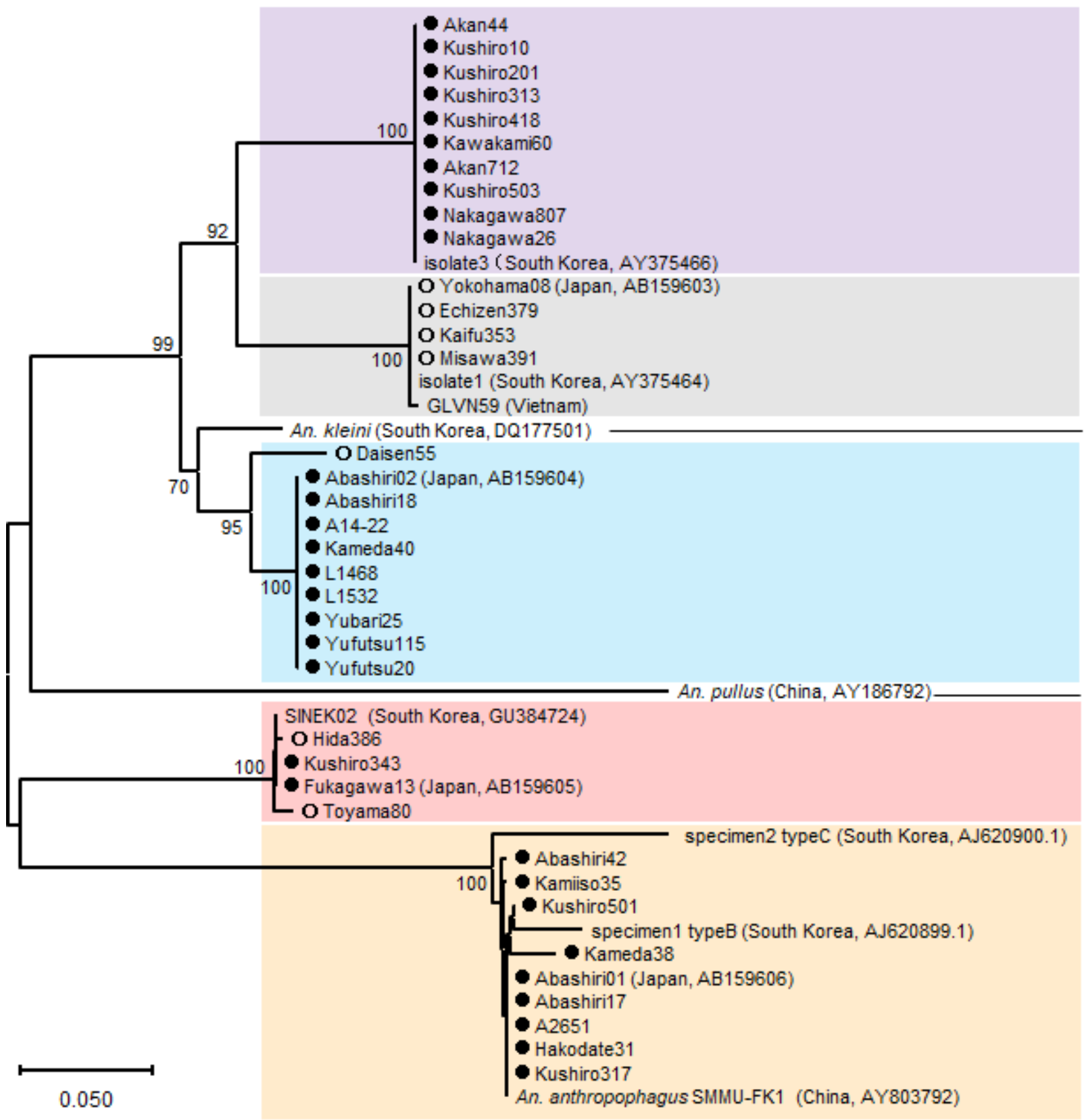

An. belenrae

An. sinensis

An. kleini

An. engarensis

An. pullus

An. sineroides

An. lesteri

\section{Figure 1}

Phylogenetic relationships among members of the Anopheles hyrcanus group. Neighbor-joining (NJ) phylogenetic tree was constructed based on partial ITS2 region nucleotide sequences. A distance matrix was calculated using the Kimura's two-parameter evolutionary model and the tree was constructed using the NJ approach in MEGA X ver. 10.2.2. The scale bar indicates the proportion of sites changing along 
each branch. The numbers on the internodes indicate percentages of 1,000 bootstrap replicates. Bootstrap values $<70$ are not shown in this figure. This phylogenetic tree is a rooted and traditional rectangular tree with An. pullus (AY186792) as an outgroup sequence. All specimens collected in the present study are marked with circles. The closed circles mark mosquito specimens collected from Hokkaido, Japan, and open circles mark specimens from the areas outside Hokkaido. Abbreviations of strains and sequence accession numbers of specimens used in this study are listed in Table 1.

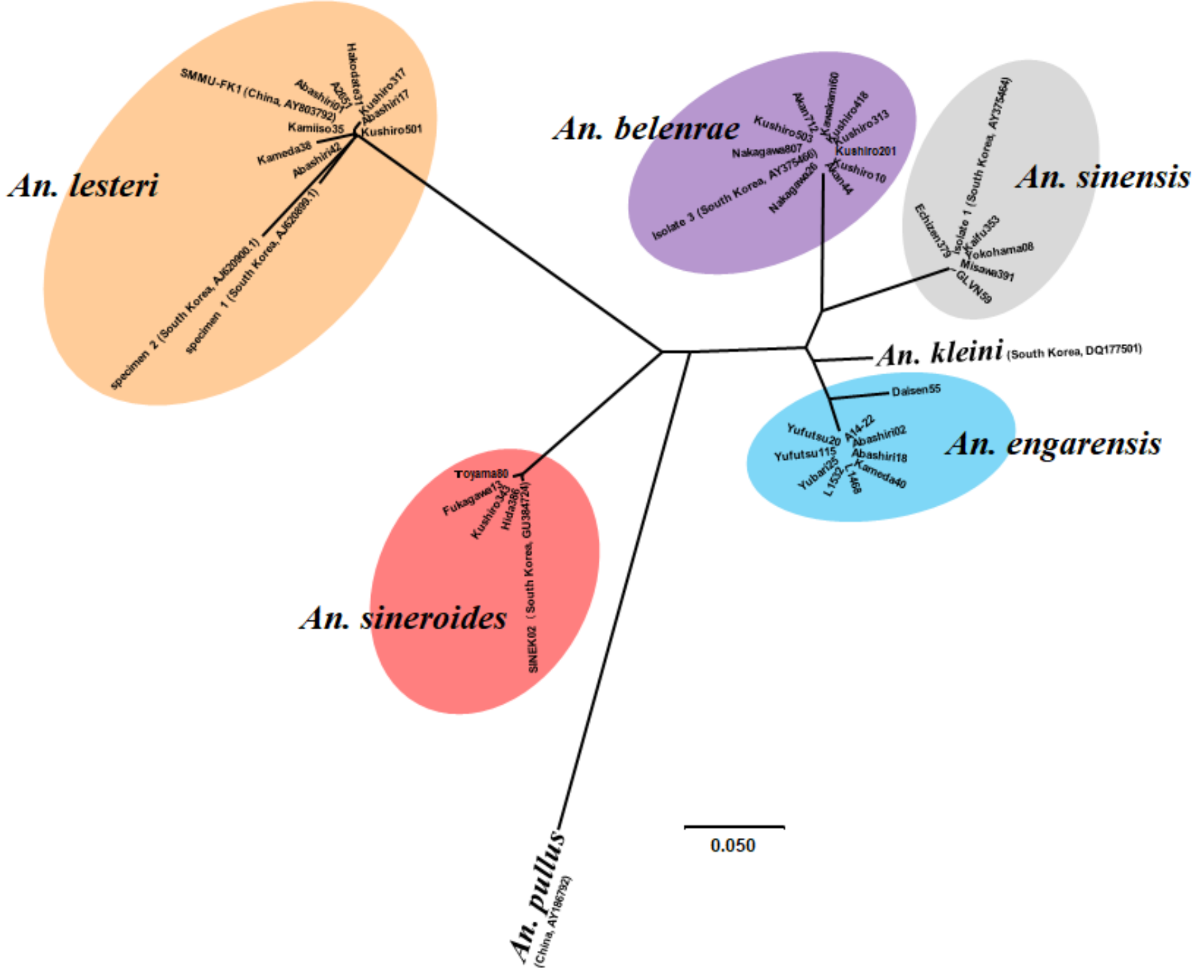

\section{Figure 2}

Phylogenetic relationships among members of the Anopheles hyrcanus group. Neighbor-joining (NJ) phylogenetic tree were constructed based on partial ITS2 region nucleotide sequences. A distance matrix was calculated using the Kimura's two-parameter evolutionary model and the tree was constructed using the $\mathrm{NJ}$ approach in MEGA X ver. 10.2.2. This phylogenetic tree is a rootless and radiation tree with An. pullus (AY186792) as an outgroup sequence to know more closely related species. The scale bar indicates the proportion of sites changing along each branch. Bootstrap values are not indicated in this 
figure. The species names in this group are bold. The members in the same species are shaded. Abbreviations of strains and sequence accession numbers of specimens used in this study are listed in Table 1.

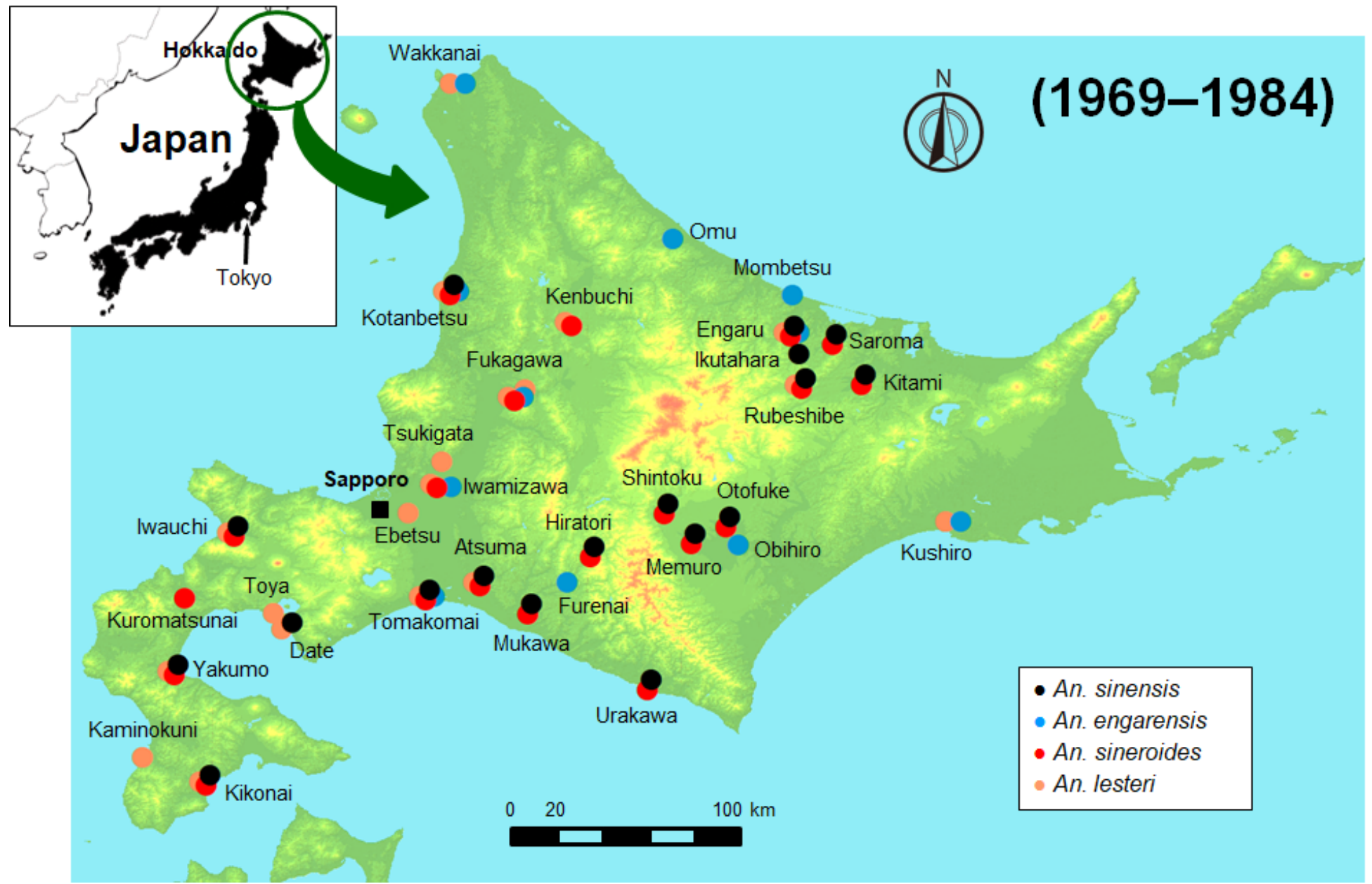

\section{Figure 3}

Distribution map of the Anopheles hyrcanus group mosquitoes in Hokkaido, Japan, as confirmed by surveys conducted during 1969-1984. This map was created from references [3-6]. This map shows mosquito species collected from 29 sites. Black, blue, red, and orange circles indicate collection sites of An. sinensis, An. engarensis, An. sineroides, and An. lesteri, respectively. 


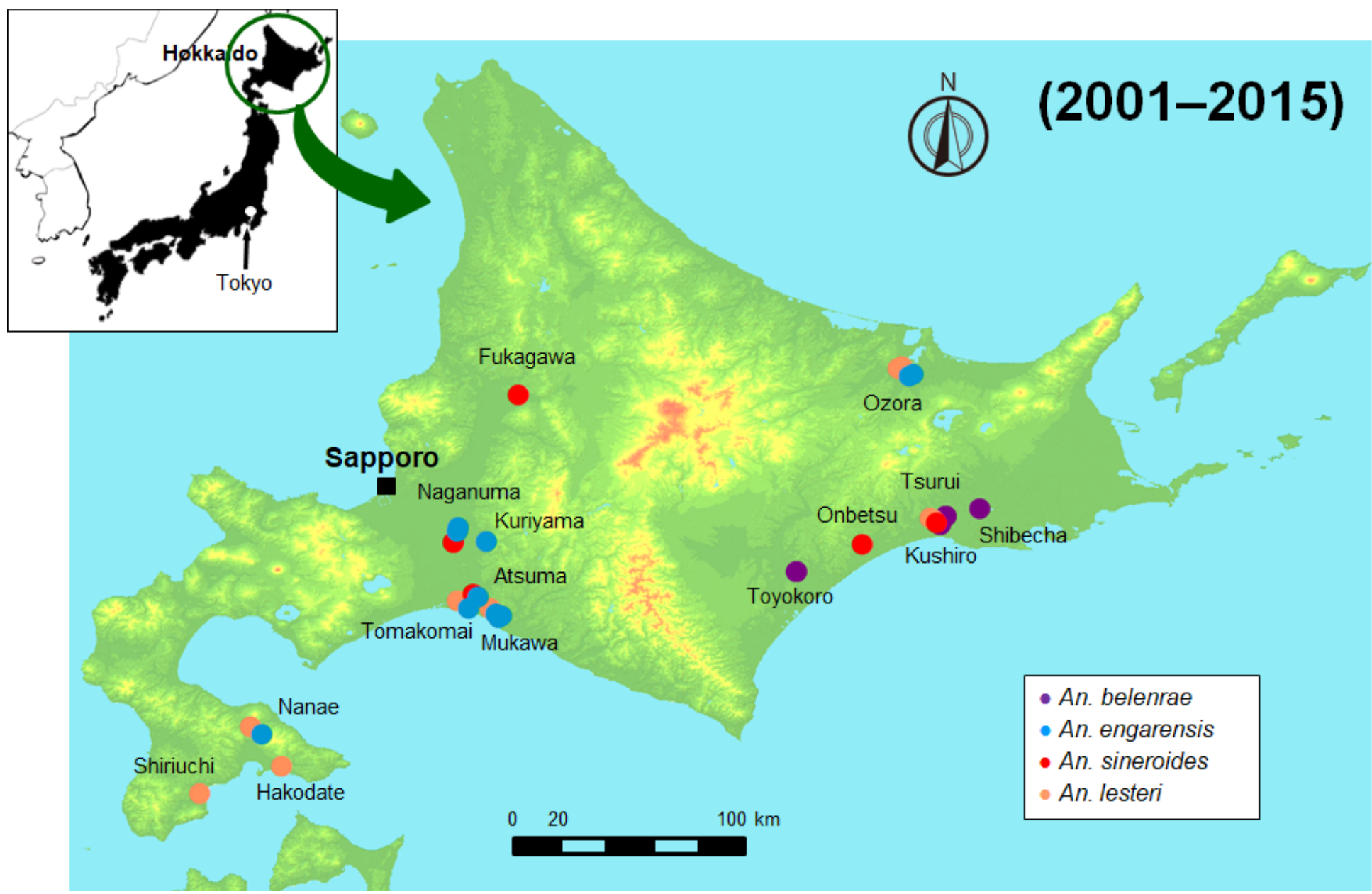

Figure 4

Distribution map of the Anopheles hyrcanus group mosquitoes in Hokkaido, Japan, as disclosed by the present study conducted during 2001-2015. This map shows mosquito species collected from 15 collection sites. Purple, blue, red, and orange circles show collection sites of An. belenrae, An. engarensis, An. sineroides, and An. lesteri, respectively.

\section{Supplementary Files}

This is a list of supplementary files associated with this preprint. Click to download.

- Graphicalabstruct.pdf

- Table1.pptx

- Table2.pptx

- Table3.pptx 